\title{
Gamificação na Educação: um modelo conceitual de apoio ao planejamento em uma proposta pedagógica
}

\author{
Leila Laís Gonçalves ${ }^{1,2}$, Graziela Fátima Giacomazzo², Flávia Rodrigeus², César \\ Bráulio Sumbo Macaia ${ }^{1}$,
}

${ }^{1}$ Laboratório de Informática Aplicada (Kiron) - Curso de Ciência da Computação Unidade Acadêmica de Ciências, Engenharias e Tecnologia (UnaCET)

${ }^{2}$ Grupo de Pesquisa em Educação e Cultura Digital - Unidade Acadêmica de Humanidades, Ciências e Educação (UnaHCE)

Universidade do Extremo Sul Catarinense (UNESC) - Av. Universitária, 1105.

Bairro Universitário - CEP: 88806-000 - Criciúma/SC - Brasil

\{llg, gfg, flaviarodrigues\}@unesc.net, brauliomacaia2@hotmail.com

\begin{abstract}
This paper presents a conceptual framework to support planning of gamification in a learning situation considering aspects of context; educational goals; skills required; behaviors and unwanted interactions; and goals -based strategies. Steps and key elements of gamification process are raised in existing models to support the proposed conceptual model.
\end{abstract}

Resumo. Esse artigo apresenta um modelo conceitual para apoiar o planejamento da gamificação em uma situação de aprendizagem considerando aspectos de contexto; objetivos educacionais; habilidades requeridas; comportamentos e interações desejados; e estratégias baseadas em objetivos. São levantados etapas e elementos fundamentais do processo de gamificação em modelos existentes para embasar o modelo conceitual proposto.

\section{Introdução}

Um dos pontos discutidos, em se tratando de escola e os jovens da atualidade, é o distanciamento que há entre a cultura escolar e a cultura da juventude. Pesquisas e experimentações buscam aproximar e incluir situações do cotidiano dos alunos no ambiente escolar. Dentre as possibilidades apresentadas tem-se o uso de jogos digitais (games em inglês, termo como são conhecidos) como ferramentas para potencializar aprendizagens em diversas áreas do conhecimento (AZEVEDO, 2012).

Nesse contexto, surge o fenômeno de gamificação (do original inglês gamification), que consiste na utilização de elementos dos jogos fora do seu contexto, com a finalidade de mobilizar os sujeitos à ação, auxiliar na solução de problemas e promover aprendizagens (KAPP, 2012). Motivados pelo crescente número de pesquisas sobre gamificação em outras áreas, esse fenômeno vem aumentado também na área da 
V Congresso Brasileiro de Informática na Educação (CBIE 2016)

Anais do XXVII Simpósio Brasileiro de Informática na Educação (SBIE 2016)

educação (LEE; HAMMER, 2012; DOMÍNGUEZ et al., 2013) como estratégia de ensino e aprendizagem. O aumento do interesse pode ser explicado, principalmente, pelo potencial da gamificação para influenciar, engajar e motivar pessoas. Contudo, para alcançar estes resultados, é imprescindível que o processo de planejamento da gamificação no contexto educativo seja realizado de forma assertiva, considerando os objetivos, conteúdos de aprendizagem, estratégias e resultados esperados.

Esse artigo apresenta um modelo conceitual para apoiar o planejamento da gamificação em uma situação de aprendizagem baseada em uma proposta pedagógica identificando etapas e elementos fundamentais do processo envolvendo: aspectos de contexto; objetivos educacionais; habilidades requeridas; comportamentos e interações desejados; e estratégias baseadas em objetivos. A abordagem da pesquisa foi qualitativa, com técnicas exploratórias buscando identificar, a partir de levantamento bibliográfico e análise de exemplos, os objetivos instrucionais cognitivos a partir da Taxonomia de Bloom e o processo de planejamento da gamificação diferenciando-o no contexto educacional. Para a análise dos dados obtidos optou-se por realizar a análise de conteúdo dos modelos e frameworks de uso geral e específico na educação visando identificar etapas e elementos de composição.

O artigo está organizado da seguinte forma: na seção 2 são apontados os principais conceitos e características que embasam a gamificação neste trabalho; a seção 3 aborda o planejamento pedagógico baseado na Taxionomia de Bloom; a seção 4 expõe modelos para gamificação que serviram de base para definição do modelo proposto descrito na seção 5; e a seção 6 apresenta as conclusões do artigo.

\section{Gamificação}

A gamificação de uma atividade refere-se à aplicação de elementos presentes em jogos como a mecânica, estética e dinâmica para engajar as pessoas, motivar ações, promover o aprendizado e solucionar problemas fora do seu contexto usual de entretenimento (KAPP, 2012).

O processo de gamificação é composto de etapas que executam diferentes atividades. Uma das atividades consiste na identificação e caracterização de elementos e procedimentos, tanto do jogo quanto do objetivo de gamificação, desenvolvida com a utilização de técnicas. As principais técnicas para a gamificação são baseadas no uso da mecânica (regras), dinâmica (comportamentos), estética (emoções) e de elementos do jogo conforme figura 1 (ZICHERMANN; CUNNINGHAM, 2011).

\begin{tabular}{|l|l|l|l|}
\hline Dinâmica de jogos & Mecânica de jogos & Estética de jogos & Elementos de Jogos \\
\hline Restrições & Regras & Motivação, Competências & Habilidades, Regras \\
\hline Progressão de níveis & Recompensa & Motivação & Pontos, medalhas, níveis \\
\hline Relações sociais & $\begin{array}{l}\text { Compartilhamento, } \\
\text { Equipe }\end{array}$ & $\begin{array}{l}\text { Engajamento, Motivação, Cooperação, } \\
\text { Colaboração, Altruísmo }\end{array}$ & Formação de equipe, Presentes \\
\hline Posição & Status, Feedback & Competição, Motivação & Ranking \\
\hline Conquista & $\begin{array}{l}\text { Desafios, Missões, } \\
\text { Vitória, Aleatoriedade }\end{array}$ & Competição, Motivação & $\begin{array}{l}\text { Desbloqueio de conteúdo, Desafios do } \\
\text { jogo, Mapas }\end{array}$ \\
\hline Personalização & Configurações & Engajamento & Avatar, Bens virtuais \\
\hline Reforço & Re-play & Competências & Loops \\
\hline Narrativa & Escolhas & Engajamento, Motivação & Diálogos, enredo \\
\hline
\end{tabular}

Figura 1. Uso da mecânica, dinâmica, estética e de elementos do jogo na gamificação.

A gamificação apresenta importante potencial no ambiente educacional, visto que sua contribuição principal se dá na motivação extrínseca e intrínseca, e no engajamento dos estudantes durante o processo de aprendizagem. As estratégias de 
V Congresso Brasileiro de Informática na Educação (CBIE 2016)

Anais do XXVII Simpósio Brasileiro de Informática na Educação (SBIE 2016)

gamificação comumente utilizadas na educação são desafios, progressão, conquista, narrativa, colaboração, recompensa, replay, experimentação (BBVA INNOVATION EDGE, 2012).

\section{Planejamento Pedagógico e a Taxonomia de Bloom}

$\mathrm{Na}$ educação, definir os objetivos de ensino e aprendizagem, significa entre outros aspectos pedagógicos, estruturar o processo pelo qual o Educador irá conduzir as atividades de estudo. A estruturação é resultado de um processo de planejamento estando diretamente relacionada "à escolha do conteúdo, de procedimentos, de atividades, de recursos disponíveis, de estratégias, de instrumentos de avaliação e da metodologia a ser adotada por um determinado período de tempo." (FERRAZ; BELHOT, 2010, p. 421). Muitos são os recursos para apoiar o planejamento pedagógico de uma disciplina, curso ou unidade de estudo, a Taxonomia de Bloom é um desses recursos teóricos, cujo papel é colaborar na definição dos objetivos ligados ao desenvolvimento cognitivo. Mesmo sendo reconhecida pelos educadores, poucos fazem uso adequado da Taxonomia de Bloom, ora por não saberem de fato como utilizá-los, ora por não se apropriarem de seus conceitos.

Neste trabalho, busca-se um planejamento pautado nos objetivos instrucionais cognitivos, definidos previamente a partir da Taxonomia de Bloom, na expectativa de que os estudantes possam alcançar altos graus de abstração. Dentre as estratégias possíveis de gamificação para tal objetivo, cita-se o uso de 'fases', conforme o jogo avança o nível de complexidade aumenta.

$\mathrm{Na}$ categorização atual da Taxonomia de Bloom, proposta por Anderson, Krathwohl e Airasian (2001 apud FERRAZ; BELHOT, 2010), a estrutura do processo cognitivo apresenta-se a partir dos verbos que identificam os objetivos: Lembrar; Compreender; Aplicar; Analisar; Avaliar; e Criar. Partindo desta revisão, Andrew Churches desenvolveu a Taxonomia de Bloom digital, objetivando incorporar novas ações (objetivos) emergentes das novas tecnologias, ou seja dos usos incorporados pelos indivíduos numa sociedade conectada. Na proposição de Churches (2014 apud MATTAR, 2014, p.86), para cada termo há um conjunto de verbos apresentados na figura 2.

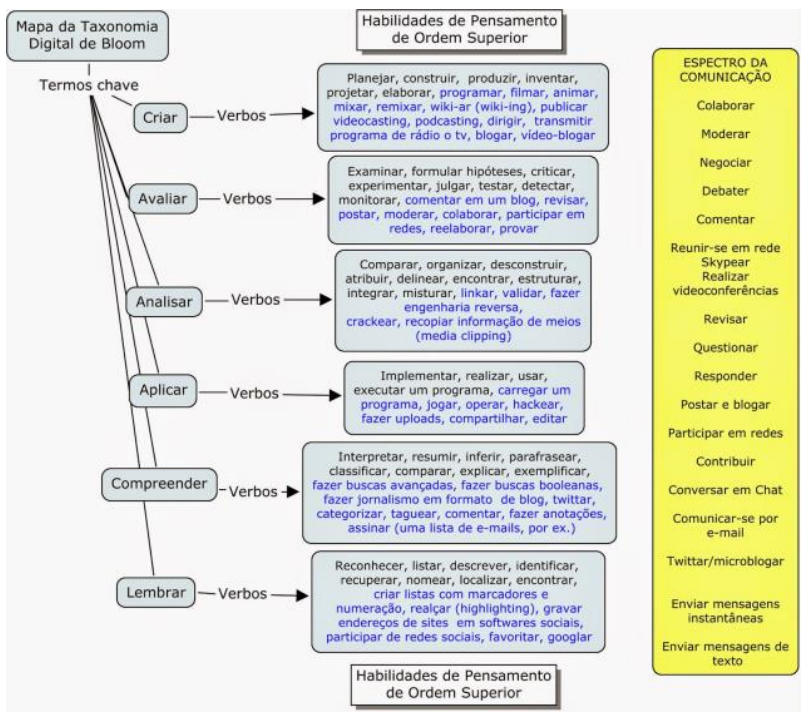


V Congresso Brasileiro de Informática na Educação (CBIE 2016)

Anais do XXVII Simpósio Brasileiro de Informática na Educação (SBIE 2016)

Figura 2. Taxonomia de Bloom digital proposta por Andrew Churches.

Esta perspectiva incentiva a pensarmos também na aplicabilidade da taxonomia em contextos gamificados, partindo dos termos chave criar; avaliar; analisar; aplicar; compreender; e lembrar; descritos aqui em ordem decrescente das habilidades do pensamento de ordem superior. É o que prospecta este estudo.

\section{Modelos para gameficação}

Os principais modelos estudados para gamificação são apresentados a seguir identificando as etapas e elementos fundamentais do processo.

Marczewski (2012) apresenta a estrutura GAME (Gather - coletar, Act - agir, Measure - mensurar e Enrich - melhorar) representando quatro etapas do processo de planejamento e construção de uma solução gamificada. Para realizar a gamificação é necessário responder a perguntas divididas nas quatro fases: planejamento, aplicação do projeto, mensuração e melhoria contínua.

De acordo com Huang e Soman (2013), o processo para a gamificação aplicada ao contexto da educação ocorre em cinco etapas: 1) compreender o público-alvo e o contexto; 2) definir objetivos de aprendizagem; 3) estruturar a experiência como o programa de aprendizagem; 4) identificar o recurso os recursos necessários para a gamificação; e 5) aplicar elementos pessoais ou sociais de gamificação (mecânicas do jogo) no programa de aprendizagem.

Em sua pesquisa, Seixas et al. (2014) apresentam quatro fases para o processo de gamificação como estratégia no engajamento de estudantes do ensino fundamental. A fase 1 visa a seleção e entendimento dos objetivos da disciplina. Na fase 2 são identificadas as ferramentas. A seleção de plataformas online de recompensa é realizada na fase 3. Por fim, a fase 4 trata da adequação das estratégias de gamificação.

O modelo conceitual proposto por Klock; Cunha; Gasparini (2015) visa auxiliar na implementação de gamificação de AVAs sendo divido em quatro dimensões tratadas em forma de questões: por quê?, quem?, o que? e como?. A respostas a estas questões objetivam mapear comportamentos presentes em AVAs, descrever os atores do sistema, identificar os dados do processo e definir os elementos de jogos a serem utilizados para incentivar determinados comportamentos.

Conforme apresentado, os principais pontos recorrentes nas propostas estudadas e relevantes para o planejamento de uma solução gamificada no contexto educacional foram: a identificação dos objetivos da gameficação, o conhecimento do público a que se destina; as estratégias e elementos do jogo a serem utilizados.

$\mathrm{O}$ diferencial desta pesquisa, em relação à grande parte dos trabalhos relacionados, está na abordagem educacional. Nesse sentido, este trabalho apresenta um modelo conceitual para apoio ao planejamento da gamificação em uma situação de aprendizagem com foco nos objetivos educacionais da Taxonomia de Bloom revisada, base da proposta pedagógica.

\section{Modelo conceitual para planejamento de gamificação na educação}

A proposição de um modelo conceitual, resultado principal da pesquisa, tem como objetivo identificar etapas e elementos fundamentais para apoiar o planejamento da gamificação em uma situação de aprendizagem. O modelo conceitual é composto de 
V Congresso Brasileiro de Informática na Educação (CBIE 2016)

Anais do XXVII Simpósio Brasileiro de Informática na Educação (SBIE 2016)

cinco etapas sendo que em cada etapa são indicados elementos do processo considerando aspectos de contexto; objetivos educacionais; habilidades requeridas; e estratégias baseadas em objetivos. A etapas e seus elementos são apresentados a seguir.

A etapa 1 consiste na Identificação dos aspectos de contexto educacional a partir do levantamento dos seguintes elementos: escola, professor, perfil dos alunos, disciplina, ano e nível escolar, turma, período, modalidade de ensino, unidade de estudo, tema, conceitos. Na etapa 2 é realizada a Descrição dos objetivos educacionais da Taxonomia de Bloom revisada. A Indicação das habilidades requeridas para o uso do ambiente gamificado é efetuada na etapa 3. São consideradas as habilidades cognitivas de pensamento de ordem superior a partir dos 5 termos da Taxionomia de Bloom revisada. A etapa 4 resulta no Mapeamento das estratégias de gamificação baseadas em objetivos educacionais. O mapeamento visa identificar e correlacionar os objetivos, conteúdo, forma de desenvolvimento dos tópicos, atividades, critérios de avaliação, recursos do jogo a serem utilizados (mecânica, dinâmica, estética e elementos), comportamentos, interações e competências socioemocionais. Para finalizar $o$ planejamento, a etapa 5 realiza a Seleção de jogos para a gamificação a partir das estratégias mapeadas.

\section{Conclusões}

O planejamento da gamificação no contexto educativo apresenta características específicas e para que seja realizado de forma assertiva deve considerar, dentre outros elementos, os objetivos, conteúdos de aprendizagem, estratégias e resultados esperados. A utilização da Taxonomia de Bloom na definição de objetivos educacionais é um importe suporte para o planejamento da gamificação e alcance de seus propósitos. Entre os trabalhos futuros, pretende-se avaliar eficácia do modelo conceitual proposto a partir de um experimento, constituindo a próxima etapa desta pesquisa já em andamento.

\section{Referências}

Azevedo, V. de A. (2012) Jogos eletrônicos e educação: construindo um roteiro para a sua análise pedagógica, In: Renote - Novas Tecnologias na Educação. UFRGS, Porto Alegre. V. $10 \mathrm{n}^{\circ} 3$.

BBVA Innovation Edge. (2012) "The fun way to engage". In: Gamification: The business of fun, p. 12-22, Madrid, BBVA Innovation Center.

Domínguez, A. et al.. (2013). Gamifying learning experiences: Practical implications and outcomes, In: Computers \& Education, 63(April), pages 380-392.

Ferraz, A. P. C. M., Belhot, R. V. (2010) "Taxonomia de Bloom: revisão teórica e apresentação das adequações do instrumento para definição de objetivos instrucionais". Gest.Prod. São Carlos, v.17, n.2, p.421-431.

Huang, W. Y.; Soman, D. (2013) “A Practitioner's Guide To Gamification Of Education". Rotman School of Management, University of Toronto.

Kapp, K. (2012) "The Gamification of Learning and Instruction: Game-based Methods and Strategies for Training and Education”. Pfeiffer. 
V Congresso Brasileiro de Informática na Educação (CBIE 2016)

Anais do XXVII Simpósio Brasileiro de Informática na Educação (SBIE 2016)

Klock, A. C. T. et al.. (2015) Um modelo conceitual para a gamificação de Ambientes Virtuais de Aprendizagem. In: Renote - Novas Tecnologias na Educação. UFRGS, Porto Alegre. V. $13 \mathrm{n}^{\circ} 1$.

Lee, J. J. Hammer, J. (2011) "Gamification in Education: What, How, Why Brother?" Academic Exchange

Quarterly, http://www.academia.edu/570970/Gamification_in_Education_What_How_Why_Bo ther, Maio.

Marczewski, A. (2012) “Gamification: A Simple Introduction”. Amazon Digital Services, Inc.

Mattar, J. (2014) "Design educacional: educação a distância na prática". São Paulo: Artesanato Educacional. Coleção tecnologia educacional;4.

Seixas, L. R. et al.. (2014) Gamificação como Estratégia no Engajamento de Estudantes do Ensino Fundamental. Mato Grosso do Sul: Anais eletrônicos do CBIE.

Zichermann, G. Cunningham, C. (2011) "Gamification by Design: Implementing Games Mechanics in Web and Mobile Apps”. Sebastopol, CA: O'Reilly Media, Inc. 